\title{
EVALUATION OF VISUALIZATION FEATURES IN THREE-DIMENSIONAL LOCATION-BASED MOBILE SERVICES
}

\author{
Mário Freitas ${ }^{1}$, A. Augusto Sousa ${ }^{1,2}$, António Coelho ${ }^{1,2}$ \\ ${ }^{1}$ DEI / FEUP, Rua Dr. Roberto Frias, s/n 4200-465 Porto, Portugal \\ 2 INESC Porto, Campus da FEUP, Rua Dr. Roberto Frias, s/n 4200-465 Porto, Portugal \\ mario.freitas@fe.up.pt,aas@fe.up.pt,acoelho@fe.up.pt
}

Keywords: Computer graphics, visualisation paradigms, location-based mobile services, visual perception, navigation systems, mobile 3D city maps, user interaction.

\begin{abstract}
Nowadays, there is a wide range of commercial LBMS (Location-Based Mobile Services) available in the market, mainly in the form of GPS-based navigation solutions, and a trend towards the display of 3D maps can be clearly observed. Given the complete disparity of ideas and a visible commercial orientation in the industry, the study of the visualisation aspects that influence user performance and experience in the exploration of urban environments using 3D maps becomes an important issue. In this work, a generic conceptual framework is proposed whose main purpose is to objectively evaluate the impact and contribution of the major visualisation elements involved (henceforth mentioned as feature vectors). With this framework in mind, an online questionnaire was developed and administered to 149 test subjects in order to measure the real impact of feature vectors. The results clearly demonstrated that certain features have clear impact on user performance, and should be taken in account in LBMS development. As an example, just by displaying buildings with a 3D appearance, subjects were able to match more accurately the real environment with the one presented on a mobile device. In general, users were able to perform the tasks entrusted to them faster, if they were provided more realistic imagery.
\end{abstract}

\section{INTRODUCTION}

The LBMS technology, namely in the form of GPSbased navigation systems, has just recently reached a state of technological maturity, enabling the development of 3D map-based graphical interfaces. Nowadays, there is a wide offer of LBMS solutions in the market, especially in the form of automotive navigation systems. Motivated by commercial interests, many of these products promise to offer the "best visualisation experience ever", in search for a differentiating factor from the competition. By looking at the variety of visualisation paradigms being proposed, one can clearly notice a great disparity of ideas without a clear notion of its usefulness.

Provided the non-existence of an objective stateof-the-art generalising theory capable of unifying and evaluating all the visualisation elements and properties, the main motivation of this work is to study the most relevant of these features and how to adjust them appropriately, in order to maximise the usability of mobile maps and to improve the navigation experience, in accordance with the following objectives:

1. Elicit and assess the state-of-the-art contributions on visualisation paradigms of 3D maps, with particular interest on mobile services and devices;

2. Develop a methodology for evaluating the different issues that influence user experience and performance when exploring an urban environment with mobile maps.

\section{STATE OF THE ART}

\subsection{Visual Perception of Realism}

The variety of free and commercial products featuring three-dimensional map-based mobile services available to the masses, usually ranges from very abstract 
to reasonably realistic and immersive visualisation paradigms. However, there is a common misconception on what is Image Realism, how is it visually perceived, and how can it be effectively measured.

In (Rademacher et al., 2001), a scientific experiment was conducted to understand what aspects of an image can make it look "real" or "not real", i.e., whether it is perceptually indistinguishable or not from the corresponding photographs. The results showed that subjects were not convinced by the increasing number of light sources and shadows nor the variety or number of shapes. The same could be said for "perfectly sharp" shadows or "perfectly polished" surfaces.

In (Lange and Ch, 2003), an experiment was carried out with 75 test subjects to classify 90 images of the virtual landscape of Brunnen / Schwyz (Switzerland) from three different viewpoints in a degree of realism from 1 (very low) to 5 (very high). The results generally demonstrated that the variable that most contributed to the sense of realism was - by far - the high-resolution orthophotographic imagery, and the second most important being texture-mapping.

In other works like (McNamara et al., 2000), the importance of perception-based image quality metrics is studied, such as the ones given by the VDP (Visible Differences Predictor) and the VDM (Visual Discrimination Metric). These two metrics aim to analytically predict the differences between a computer-generated image and the photograph it depicts, taking into account the limitations of the human eye described by the HVS (Human Visual System). The VDP quality metric takes the two images as input and generates a difference map that predicts the probability of the human eye finding differences between the two pictures, as demonstrated in (Bolin and Meyer, 1999) (see Figure 1).

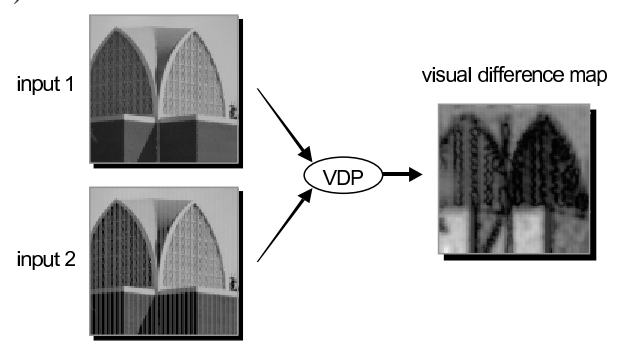

Figure 1: Difference map in the VDP quality metric (obtained from the previously mentioned work).

A simplification of the VDM quality metric was provided by following a similar approach (Bolin and Meyer, 1999): instead of finding a difference map, a just noticeable difference map was proposed which corresponds to a $75 \%$ probability of a person detecting a difference between the two images (McNamara et al., 2000).
Because of some controversy and no agreedupon standards for measuring realism in computergenerated imagery, a conceptual framework for measuring image realism and evaluating its usefulness was proposed in (Ferwerda, 2003). The framework distinguishes three different varieties of realism: physical realism, photo-realism and functional realism. However, this framework does not seem to be enough to encompass the extents to which reality or virtuality can be "augmented". Accounting for such circumstances, the concept of Virtuality Continuum was introduced in (Milgram and Kishino, 1994) as represented in Figure 2.

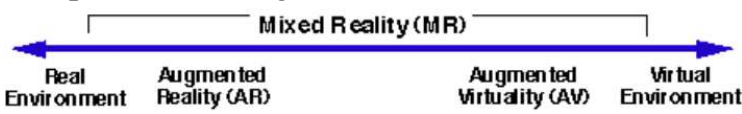

Figure 2: The Virtuality Continuum (obtained from the previously mentioned work).

At the left end, we have the "completely real" Real Environment, which is made up of "real" objects: "any objects that have an actual objective existence". At the right end, we have the "completely computersimulated" Virtual Environment, which is made up of "virtual" objects: "objects that exist in essence or effect, but not formally or actually".

\subsection{User tasks}

The underlying basic equation that can help us find the "perfect" balance in map-based mobile services is what could be called of Mobility Equation. This equation was first formulated by Leonard and DurrantWhyte for mobile robot navigation (Borenstein et al., 1996) but can be equally extended to human navigation. The equation is made up of the following three questions:

- 'Where am I?'

- 'Where am I going?'

- 'How do I get there?'

In (Hunolstein and Zipf, 2003), the tasks are classified into 4 different groups of high-level user tasks that have a strong relationship with these questions, as described in table 1 .

\subsection{Location-Based Mobile Services}

In this work we have analysed and studied several state-of-the-art contributions on LBMS which provide a wide variety of visualisation paradigms, in order to understand the current tendencies in the industry and to formulate hypothesis regarding their validity and usefulness. The contributions range from pilot studies to commercial products, within the scope of 
Table 1: The primary tasks that 3D maps are used for.

\begin{tabular}{|l|l|}
\hline Task & Description \\
\hline Locator & $\begin{array}{l}\text { Identification of the user's own po- } \\
\text { sition and other objects. Answers } \\
\text { 'Where am I?' questions. }\end{array}$ \\
\hline Proximity & $\begin{array}{l}\text { Inform the users of nearby facili- } \\
\text { ties. Implied by 'Where am I go- } \\
\text { ing?' questions. }\end{array}$ \\
\hline Navigation & $\begin{array}{l}\text { The most tangible example is rout- } \\
\text { ing from one location to another. } \\
\text { Answers 'How do I get there?' } \\
\text { questions. }\end{array}$ \\
\hline Event & $\begin{array}{l}\text { Time/Location dependent objects, } \\
\text { allowing the users to know what is } \\
\text { happening and when/where. An- } \\
\text { swers 'And now what?' questions. }\end{array}$ \\
\hline
\end{tabular}

road and pedestrian maps, as follows: TellMaris, mLOMA, LAMP3D, TomTom, Navigon, NDrive, iGO, Google Earth, INSTAR, Virtual Cable ${ }^{\mathrm{TM}}$ and Enkin.

\section{CONCEPTUAL FRAMEWORK}

In this section, a generic evaluation framework is proposed which can be used as the main methodology for the specification, development and evaluation of new or existing solutions in the visualisation problem domain. This framework is proposed in order to simplify the evaluation process to the most relevant features, to the detriment of other classical analysis methods that can be used to obtain a more thorough evaluation. This framework defines the concept of feature vectors comprising orientations and magnitudes. The orientation defines the idea or concept the visualisation paradigm represents, and magnitude the degree/level to which the paradigm "amplifies" the vector. An example can be seen in Figure 3 to describe a possible feature vector for transportation. An orientation of this feature vector is the mode of transport, while pollution, cost and speed are magnitudes.

The framework is composed by six feature vectors as shown in Figure 4 and described below. These feature vectors are not intended to characterise the complete set of visualisation features, but the most relevant ones observed from the current state of the art described in section 2.3.

\subsection{Image Realism}

Image Realism is the feature vector that is concerned with how real, i.e., free from any idealisations or abstractions, is the image of the map presented to the user. Taken into account what was previously

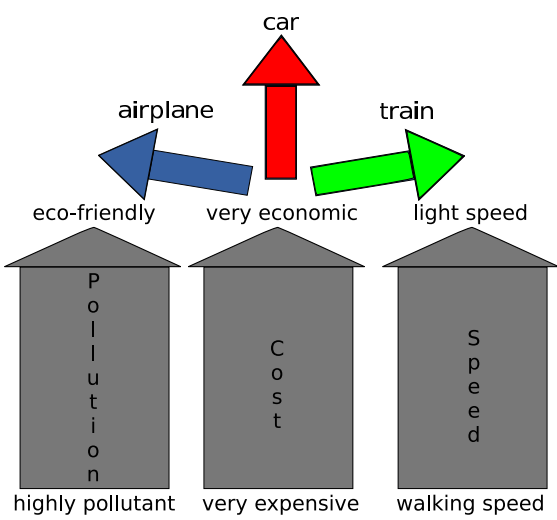

Figure 3: A possible feature vector for "Transportation".

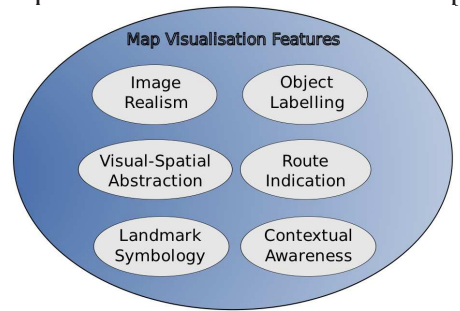

Figure 4: Evaluation Framework through feature vectors.

mentioned on this matter (see Section 2.1), the suggested magnitudes for this vector will be based on the framework proposed in (Ferwerda, 2003) and the concepts on virtuality continuum defined in (Milgram and Kishino, 1994), with a few modifications. Firstly, a "relaxed" version of physical realism will be adopted, i.e., it is assumed that current displays are considered perfect in the sense that they can emit the actual energy we want them to reproduce. Secondly, this framework will be incorporated into the virtuality continuum as illustrated in Figure 5, adapted from the above work.

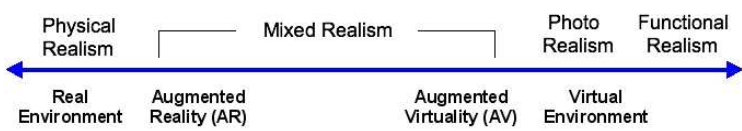

Figure 5: An illustration of the proposed framework combining the Virtuality Continuum spectrum with varieties of image realism (adapted from the previously mentioned work).

Photo-Realism is located to the left of Functional Realism, not because it is considered "less virtual" than Functional Realism but because it is closer to the Physical Realism, and consequently providing a more "realistic" environment.

In terms of orientations, this vector includes the visualisation elements that represent the real world visual information, namely $3 D$ Buildings (city buildings, landmarks), Map Vectors (roads and polygons), and Surface Model (ground surface elevations). 


\subsection{Object Labelling}

Object Labelling encompasses the kind of visual techniques and strategies that are followed to label map elements such as rivers, streets, cities, and so on.

In (Been et al., 2006) and other studies, the importance of two types of labelling, namely static labelling and dynamic labelling, is discussed. This is relevant to distinguish since, depending on the case, we might be dealing with dynamic maps, i.e., maps that support continuous zoom (changing the scale) and continuous panning (usually by dragging the map). Based on the framework proposed in the previous study, the magnitudes for this vector will include the concepts of Static/Dynamic Selection (visibility) and Placement (size, position and orientation) of labels.

One of the possible approaches when labelling objects is to project the labels oriented towards the current perspective, analogous to a billboard in Computer Graphics. This approach is followed by all the contributions except Google Earth where labels are flattened and laid down on the maps surface.

Based on the works of (Wolff, 1999; van Dijk et al., 1999) and the previous discussion on adaptiveness to the current perspective, the proposed orientations for Object Labelling are Perspective-Adaptive (oriented towards the current perspective), Point Positioning (point symbols), Line Positioning (polygonal chains, such as rivers), Area Positioning (areal features such as countries), and General Positioning (a combination of the three previous methods).

\subsection{Visual-Spatial Abstraction}

Visual-Spatial Abstraction measures the complexity of mental operations that are required to perform the visual matching of the real environment that can be observed and the one on the screen. This vector is specifically focused on the mental viewing transformation that is required in order to have a perfect correspondence between both images: the reality and the screen. The proposed orientations for this vector are presented, regardless of the elevation angle of the "camera", namely Ground Level (when it is only possible to observe the current street and its junctions), Local-Area Level (when streets that may not even be part of the route can be observed), and Wide-Area Level (when municipalities and an overview of the route are visible). The proposed magnitudes reflect the adaptiveness of the camera to the users' behaviour. We define Adaptive Level and Adaptive Orientation when the camera adapts to the user's movement (according to some variable like speed), and whether it adapts to his looking direction, respectively.

\subsection{Route Indication}

Route Indication provides a classification of the visual techniques and strategies for showing the itinerary path in the road maps, and the kind of manoeuvre indicators or way points that are presented in the display. The proposed orientations for this vector, can be regarded as the visual indicators that are generally used by the majority of the contributions to display the route, namely Arrows, Cords, Way Points and Carpet-like shapes to indicate the route. These indicators can be used with different "immersion" levels which are considered the proposed magnitudes for Route Indication, namely Instructive (when indicators are merely instructive) and Simulative (when they resemble real world indicators).

\subsection{Landmark Symbology}

Landmark Symbology evaluates the cartographic symbology that is used to portray the world using a pictorial language, represented by "map symbols", often accompanied by a legend. This vector is also related to Image Realism, in the way that both should be complementary, i.e., excessive realism may distract the users, but a great lack of symbology may completely blur their sense of orientation.

New concepts and design guidelines for the cartographic visualisation of landmarks in mobile maps are proposed in (Elias et al., 2005). Based on these concepts, the orientations for this vector will reflect the kind of buildings represented by symbols, specifically Shops referenced by name (e.g., KFC, McDonalds), Shops referenced by type (e.g., hotel, pharmacy), Buildings with unique name / function (e.g., Tokyo Tower, Statue of Liberty), and Buildings with unique visual properties (e.g., "the large yellow house"). Additionally, the first proposed magnitude for this vector will define in itself, the concept of levels of abstractions for landmarks, according to a scale (from the most abstract, to the most concrete): Words, Sign, Icon, Sketch, Drawing, and Image, as defined in the previous study.

There are other parameters that influence the decision of whether an abstraction level should be used in a mobile map for a given situation. For instance, some cartographic generalisation procedures (like scaling down a landmark object to an appropriate size suited for its representation in a map) might raise some problems such as congestion, coalescence, and imperceptibility (Elias et al., 2005). To account for these restrictions, the proposed magnitudes consist of Adaptive Zoom and Adaptive Complexity, respectively, whether the abstraction level of landmarks 
adapts to the current zoom level, and whether they change with the varying complexity of features.

\subsection{Contextual Awareness}

Contextual Awareness measures the extent to which a visualisation paradigm is applied to get additional information on a contextual or situational basis.

It is important to distinguish the three groups of application areas in which virtual urban environments can be valuable, according to the spatio-temporal nature. These groups constitute the proposed orientations for this vector, depending on whether they focus on the past, present or fiction, according to (Coelho, 2006): Reconstructional (reconstruction of urban environments that were totally or partially lost), Recreational (urban design, urban planning, etc.), and Fictional (creation of imaginary realities).

Levels of awareness regarding the current location, time, and situation can vary from contribution to contribution. In (Burigat and Chittaro, 2005), it is claimed that a passive contextual-awareness approach is generally more flexible than an active approach. In the latter case, if the user is constantly presented with unwanted information it can become "too obtrusive". Contrarily, in most automotive navigation systems, direction instructions or location-based information such as nearby points of interest are automatically presented, i.e., without the need of the user's intervention. For these reasons, the proposed magnitudes for this vector will reflect the different autonomy levels of "contextual awareness" an application can demonstrate in different contexts and tasks, as previously denoted by (Chen and Kotz, 2000), specifically Active Awareness (without the need of user intervention), and Passive Awareness (when the user shows interest for getting context-based information).

Table 2 summarises the evaluation framework, according to the proposed magnitudes and orientations.

\section{METHODOLOGY}

An interactive online questionnaire was developed and several hypothesises were formulated, in order to assess the real impact of each visualisation feature described in the conceptual framework. Since available free online questionnaires are generally limited to allow users to set their preferences, an interactive online questionnaire was developed specifically for this study, enabling the measuring of time for each answer and a more adequate visual aspect definition. However, due to the intrinsic limitations of the proposed questionnaire, and in order not to make it per- ceived by potential participants as "too exhaustive", only the features for which there are no significant indications from the state-of-the-art (regarding their impact and relevance) were evaluated with the questionnaire. Moreover, there are some components that were not possible to evaluate, and therefore were not included in this study, given the limitations imposed by this kind of questionnaire.

The questionnaire was divided into 3 parts. In the first part, the exercises were mainly based on the pointing task paradigm as previously performed in other studies (Nurminen, 2006). In the second part, a similar approach was followed, but instead of evaluating the matching of the two realities, the main objective was to measure how well users perform a given task (see Section 2.2). In the last part, users were asked about their preferences regarding the visualisation of map elements such as landmarks.

\subsection{Image Realism}

All Image Realism orientations were tested along with the various degrees of magnitudes, in accordance with the vector instances (orientations and magnitudes combined) found in the state-of-the-art contributions. These instances were considered eligible for the evaluation through the questionnaire, since there are few or no indications, with regards to their impact:

- Simple Textured Buildings and Photo Textured Buildings

- Coloured Map and Orthophotomap

\section{- Flat Model and Terrain Model}

It was hypothesised that, in the absence of Simple Textured Buildings, test subjects will have to rely on their ability to match the 3D geometry of the real building with the geometry of the $2 \mathrm{D}$ polygon representation on the map. At the same time, it is supposed that by providing the three-dimensional (yet simple) geometry of the whole building, in the presence of this component, test subjects will make fewer mistakes and, as a consequence, will require less time matching both realities (see Figure 6).

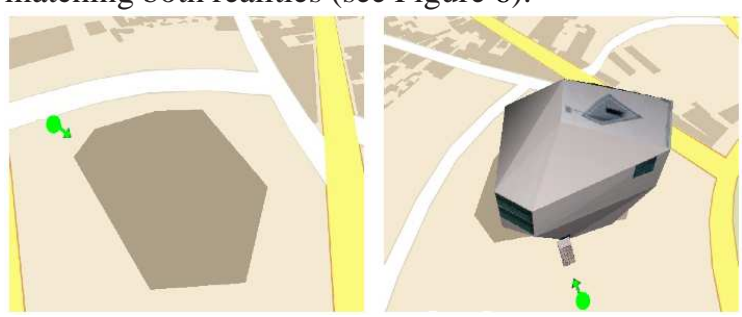

Figure 6: The 2 images supporting the questions that evaluate the impact of Simple Textured Buildings.

In the case of the Photo Textured Buildings component (see Figure 7), it was hypothesised that, by 
Table 2: Structure of the proposed evaluation framework.

\begin{tabular}{|l|l|l|}
\hline Feature Vector & Orientations & Magnitudes \\
\hline Image Realism & $\begin{array}{l}\text { 3D Buildings, Map Vectors, Surface } \\
\text { Model }\end{array}$ & $\begin{array}{l}\text { Physical Realism, Mixed Realism, } \\
\text { Photo-Realism, Functional Realism }\end{array}$ \\
\hline Object Labelling & $\begin{array}{l}\text { Perspective-Adaptive, Positioning } \\
\text { (Point, Line, Area, General) }\end{array}$ & $\begin{array}{l}\text { Static / Dynamic Selection / Place- } \\
\text { ment }\end{array}$ \\
\hline Visual-Spatial Abstraction & $\begin{array}{l}\text { Ground Level, Local-Area Level, } \\
\text { Wide-Area Level }\end{array}$ & Adaptive Level, Adaptive Orientation \\
\hline Route Indication & Arrows, Cords, Way points, Carpet & Instructive, Simulative \\
\hline Landmark Symbology & $\begin{array}{l}\text { Shops (referenced by name), Shops } \\
\text { (referenced by type), Buildings (with } \\
\text { unique name / function), Buildings } \\
\text { (with unique visual properties) }\end{array}$ & $\begin{array}{l}\text { Abstractness (Words, Sign, Icon, } \\
\text { Zoom, Adaptive Complexity }\end{array}$ \\
\hline Contextual Awareness & $\begin{array}{l}\text { Reconstructional, Recreational, Fic- } \\
\text { tional }\end{array}$ & Active Awareness, Passive Awareness \\
\hline
\end{tabular}

simultaneously providing the 3D geometry of a building along with photographic façades, test subjects will be able to detect features (e.g. windows, doors, unique wall patterns, etc.) more accurately and faster than in the case of Simple Textured Buildings.

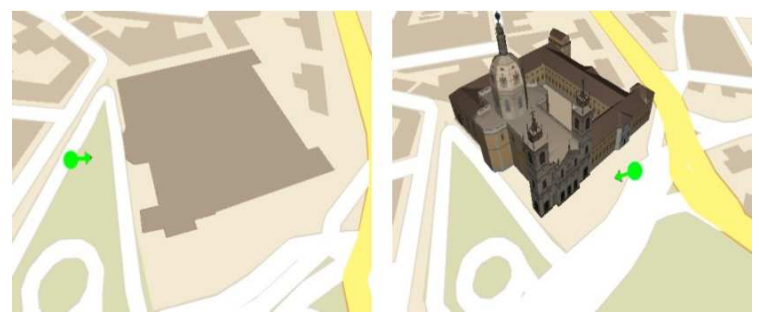

Figure 7: The 2 images supporting the questions that evaluate the impact of Photo Textured Buildings.

Regarding Map Vectors, it is assumed that an $\mathrm{Or}$ thophotomap can provide subjects a much more enriching visualisation experience than the one provided by a Coloured Map (see Figure 8). The hypothesis rests on the belief that an Orthophotomap component can make easier for users to discern the true features of the map's surface, by giving a realistic view rather than a rough generalisation. There are many situations were coloured vector polygons are not enough to represent features like a tiled pavement; a group of trees arranged in a special and unique way; and several "static" features like public benches, zebra crossings, and many others that are impossible to find in a coloured vector map.
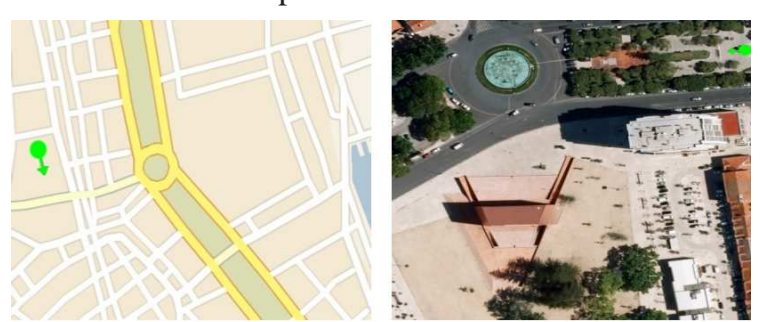

Figure 8: The 2 images supporting the questions that evaluate the impact of Coloured Map and Orthophotomap.
Regarding the Surface Model, it was hypothesised that by using a Terrain Model rather than a Flat Model component, users will be able to perform the spatial matching of both reality and virtuality in a much more immersive and natural way (see Figure 9). It is expected that by providing the Terrain Model component, users will be able to use elevated reference points, and to understand and visualise occlusions caused by the varying landscape elevation.

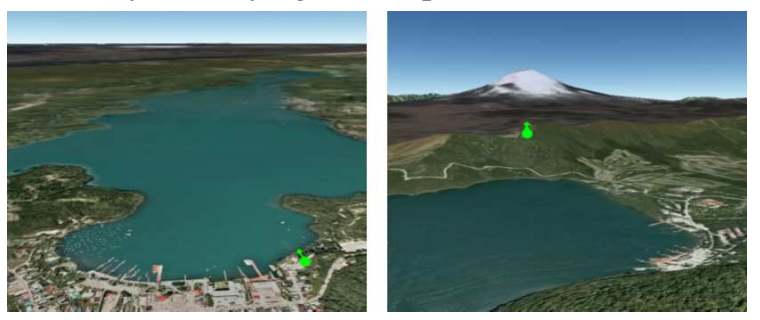

Figure 9: The 2 images supporting the questions that evaluate the impact of Flat Model and Terrain Model.

In the end, it is expected that users will be able to perform their tasks in less time, since they just need to think "outside the box". On the other hand, by using a Flat Model, users would understand that the image on the screen does not account for occlusions, and therefore, they would have to do that job themselves.

\subsection{Object Labelling}

With respect to Object Labelling, it was hypothesised that, when users are analysing labels (e.g. of streets, rivers, cities, and so on) which are not oriented towards the current viewing direction depicted in the device, they will feel much more difficulty reading the words, due to the decreased visibility, especially when looking in a direction which is parallel to the map's surface (see Figure 10).

In such case, users will not be able to read labels as faster, and will pan the map closer to the camera so 


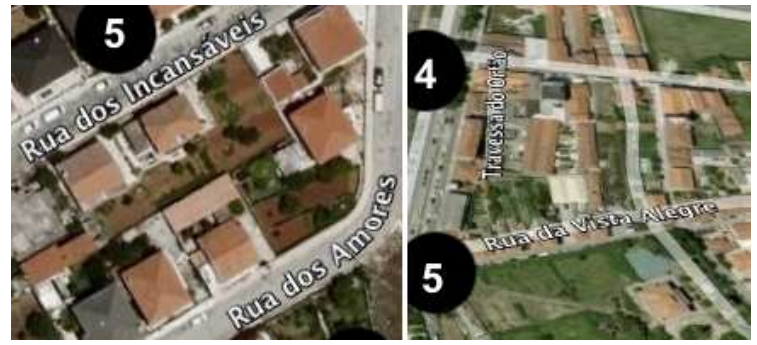

Figure 10: The 2 tasks that evaluate the impact of Perspective-Adaptive Labelling (close-up).

it becomes easier to read. Particularly in the case of labels which are almost parallel to the camera's viewing direction, some users will wish to skip words, if they find them "too difficult" to read.

\subsection{Route Indication}

It was hypothesised that, when a user is presented with an image which looks more familiar to him, given the current context, the user will be able to perform his task with lesser effort (see Figure 11). It is assumed that users won't make more mistakes using one approach or the other, but that a significant difference in the time they require to complete their task may arise, i.e., that a Simulative component will result in faster responsiveness than an Instructive approach.

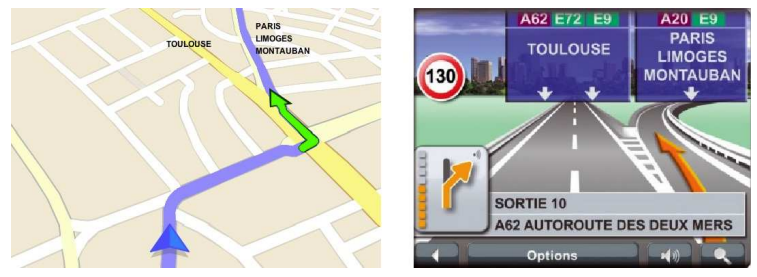

Figure 11: 2 of the tasks that evaluate the impact of Instructive and Simulative route indications.

\subsection{Landmark Symbology}

For this feature vector, it was hypothesised that users will require Adaptive Zoom functionality, i.e., that the majority of them will choose an abstract landmark representation of a given building, when a map which is zoomed out far from the ground is used, but a more concrete representation when at close range (see Figure 12).

The basis of such hypothesis rests on the various issues raised by the cartographic generalisation procedures, as previously explained in Section 3.5. For instance, even if a concrete landmark is used rather than an abstract representation, there are certain zoom levels of a map which do not allow users to perceive enough features of that landmark, in order to identify it with a significant confidence level.
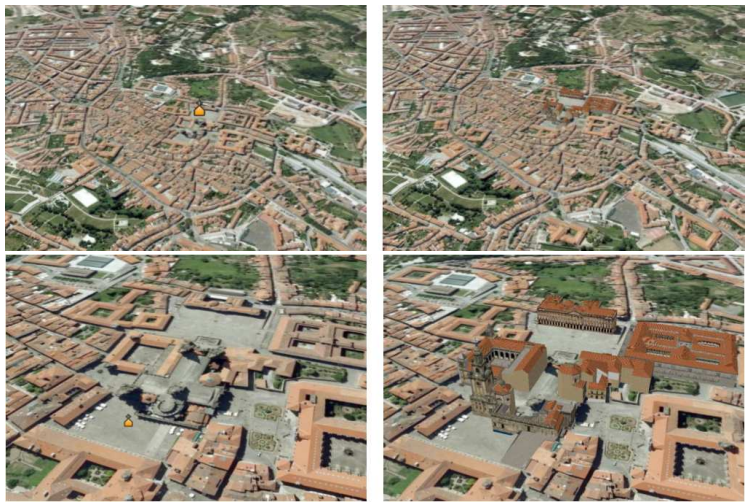

Figure 12: The preferences that evaluates the users' need for an Adaptive Zoom approach, when a map which is zoomed out far / zoomed in close to the ground is used.

\section{RESULTS}

In total, 149 test subjects answered the questionnaire, mostly from a student population in Computer Science and Informatics: $89 \%$ were male, and $78 \%$ were in the 18 to 25 age group. In general, prior to answering the questionnaire, subjects considered themselves fairly capable of using both maps and GPS navigators, given the approximate 50-50 ratio shared between "average" and "experienced" users. Only 3\% of the participants reported they were unfamiliar with either maps or GPS navigators.

\subsection{Image Realism}

Regarding the impact of the presence and absence of Simple Textured Buildings, there were $91 \%$ and $77 \%$ correct answers, respectively, in both situations. Although slight, the difference between the two cases shows the advantage of the presence of Simple Textured Buildings over its absence. Test subjects required, in average, $11 \mathrm{~s}$ ( $7.3 \mathrm{~s}$ standard deviation) to answer when buildings were shown, proving to be faster than when using a classic $2.5 \mathrm{D}$ map (avg. $15 \mathrm{~s}, 6.4 \mathrm{~s}$ s.d.). While in this case there was just a $14 \%$ difference in the number of correct answers, in the case of Photo Textured Buildings component there were $88 \%$ and $30 \%$ correct answers respectively. Despite this difference between both questions, the number of correct answers in the presence of Photo Textured Buildings was almost the same as in the case of Simple Textured Buildings. In terms of answers times, $95 \%$ of the subjects had already answered before the first 21s in the presence of Photo Textured Buildings, about $4.4 \mathrm{~s}$ less than in the presence of Simple Textured Buildings. When the buildings were all removed from the exercise with Photo Textured Buildings (i.e., in its absence), $95 \%$ of test subjects answered before the first $42.7 \mathrm{~s}$ (avg. 17.8s, s.d. 14.4s) against 25.4s (avg. 
$15 \mathrm{~s}$, s.d. $6.4 \mathrm{~s})$. This clearly demonstrates that the results with Photo Textured Buildings are more stable, considering the increase in difficulty of the exercise.

In the presence of a Coloured Map, the number of participants who were unable to answer the question was quite high (14\%). The same happened with the number of wrong answers being quite different from the Orthophotomap (67\% and 7\%, respectively). Nevertheless, subjects had no apparent difficulty in finding the correct answer, in the presence of the Orthophotomap component, as $92 \%$ chose the correct answer in similar conditions (as shown in Figure 13). Besides being more effective, the Orthophotomap proved also to be more efficient, as subjects took an average time of $9.3 \mathrm{~s}$ (s.d. 18.4s) to answer the question, considerably faster compared to the $23.5 \mathrm{~s}$ (s.d. 16.8s) in the case of the Coloured Map.
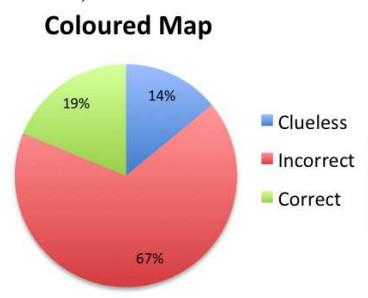

\section{Orthophotomap}

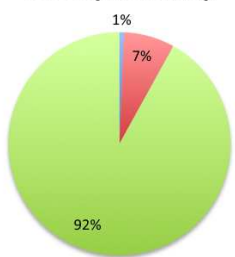

Figure 13: Answers in the presence of a Coloured Map and an equivalent Orthophotomap.

In terms of Surface Model, there was just a 5\% difference in the number of correct answers between both cases, with advantage to the Terrain Model. However, the Terrain Model was much more efficient, as the average response time was $7.5 \mathrm{~s}$ (s.d. $5 \mathrm{~s}$ ), compared to the 15.3 s (s.d. 13.8s) obtained with the Flat Model.

These results point out that image realism can improve the task of matching the 3D map with reality, both maximising effectiveness (lesser mistakes) and effectiveness (lesser time).

\subsection{Object Labelling}

With respect to Object Labelling, when labels were oriented towards the camera, the subjects took lesser time to perform the task (avg. 11.8s, s.d. 5.2s) than when labels were not oriented according to the camera (avg. 15s, s.d. 6.4s), as shown in Figure 14.

From these results a conclusion can be made that Perspective-Adaptive Labelling can increase readability of labels in 3D maps.

\subsection{Route Indication}

With respect to the Route Indication there was no relevant difference in terms of answer correctness between Instructive or Simulative components. How-

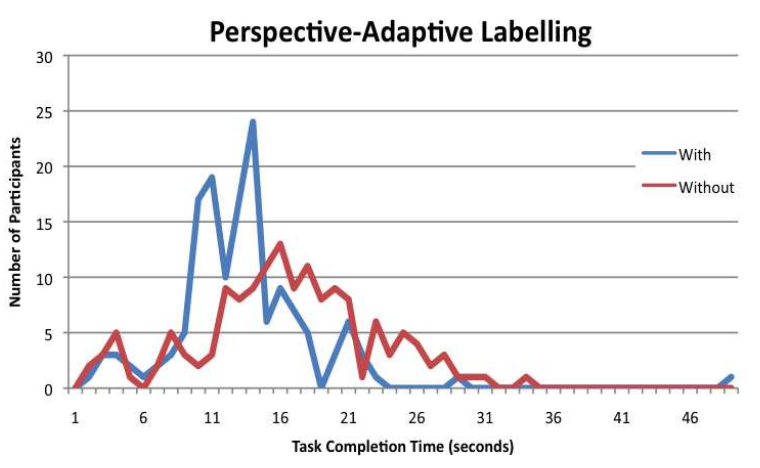

Figure 14: Answer times in the presence and absence of Perspective-Adaptive Labelling component.

ever, in terms of efficiency, the Simulative component resulted in faster response times (avg. 8.6s, s.d. 5.9s), against (avg. 11.8s, s.d. 7.9s) in the case of the Instructive component.

Although both techniques can achieve similar levels of correctness, the Simulative approach can speedup the task of matching reality with the 3D map. This can be of great importance when supporting activities that demand short response times, such as driving.

\subsection{Landmark Symbology}

A vast majority of participants (87\%) answered they would more easily identify and recognise the presence of a given distant landmark, when an abstract representation of that landmark was used. Approximately $86 \%$ of them indicated their preference towards the use of concrete landmarks at close range.

Different zoom levels over 3D maps will encompass also different levels of visual complexity, and as such, Adaptive Zoom functionality is of great importance for maximising readability.

\section{CONCLUSIONS AND FUTURE WORK}

In this study, a generic Evaluation Framework was proposed as the main methodology for the specification, development and evaluation of new or existing solutions in the 3D map visualisation problem domain for LBMS. Feature Vectors can individually describe a set of choices (orientations) and degrees of applicability (magnitudes). The proposed framework focuses on 6 feature vectors namely, Image Realism, Object Labelling, Visual-Spatial Abstraction, Route Indication, Landmark Symbology, and finally Contextual Awareness. These feature vectors encompass the most relevant visualisation issues in $3 \mathrm{D}$ maps on LBMS, but there was no intent to cover them completely. A future line of research would consist in 
analysing the totality of features that address visualisation aspects, in the context of exploration of urban environments, using 3D LBMS as guidance.

Although the state of the art contemplates some of the issues involved, the questionnaire gave a much more clear insight on them. In general, it is observed a greater tendency towards the need of Image Realism rather than Image Functionalism. In terms of Perspective-Adaptive Labelling, it was proved that users are at disadvantage, if they are given the task to read labels of a map, when these labels are not oriented towards the camera's viewing direction. The results also demonstrated that users can more easily identify the presence of a distant landmark with an abstract representation, and a close landmark with a concrete representation, which is indicative of the need of an Adaptive-Zoom behaviour.

Since there are several limitations on the kind of measurements that can be performed with the proposed questionnaire in order to evaluate feature vectors, it would be interesting to perform other kinds of tests, with particular focus on dynamic experiments, to get more information about other vectors such as Visual-Spatial Abstraction and Contextual Awareness which were not evaluated. An example of these experiments would include using a driving simulator to test the participants' reflexes, given a situation where they are approaching a manoeuvre, and deciding which way to go.

From the results obtained from this work, and future lines of research, we expect the definition of new paradigms of visualisation for 3D map visualisation on LBMS that maximise usability and improve user experience and performance.

\section{ACKNOWLEDGEMENTS}

We would like to thank NDrive Navigation Systems, $S . A$. for the support provided for this research project.

\section{REFERENCES}

Been, K., Daiches, E., and Yap, C. (2006). Dynamic map labeling. IEEE Transactions on Visualization and Computer Graphics, 12(5):773-780.

Bolin, M. R. and Meyer, G. W. (1999). A visual difference metric for realistic image synthesis. In Proc. SPIE, pages 106-120.

Borenstein, J., Everett, H. R., and Feng, L. (1996). ”Where am I?" - Sensors and Methods for Mobile Robot Positioning. The University of Michigan.
Burigat, S. and Chittaro, L. (2005). Location-aware visualization of vrml models in gps-based mobile guides. In John, N. W., Ressler, S., Chittaro, L., and Duce, D. A., editors, Web3D, pages 57-64. ACM.

Chen, G. and Kotz, D. (2000). A survey of contextaware mobile computing research. Technical Report TR2000-381, Dept. of Computer Science, Dartmouth College.

Coelho, A. (2006). Expeditious Modelling of Virtual Urban Environments based on Interoperability and Geospatial Awareness (in Portuguese). $\mathrm{PhD}$ thesis, Faculdade de Engenharia da Universidade do Porto.

Elias, B., Paelke, V., and Kuhnt, S. (2005). Concepts for the cartographic visualization of landmarks. In Proceedings of Symposium 2005 Location Based Services \& Tele Cartography, page 11.

Ferwerda, J. A. (2003). Three varieties of realism in computer graphics. In In Proceedings SPIE Human Vision and Electronic Imaging '03, pages 290-297.

Hunolstein, S. V. and Zipf, A. (2003). Towards task oriented map-based mobile guides. In Workshop "HCI in Mobile Guides" at Mobile HCI 2003. 5th International Symp. on HCI with Mobile Devices and Services.

Lange, E. and Ch, Z. R. (2003). The degree of realism of GIS-based virtual landscapes: Implications for spatial planning. In D. Fritsch and R. Spiller (eds) Photogrammetric Week '99, pages 367-374.

McNamara, A., Chalmers, A., and Trocianko, T. (2000). Visual perception in realistic image synthesis. In Coquillart, S. and Duke, D., editors, STAR Proceedings of Eurographics 2000, Interlaken, Switzerland. Eurographics Association.

Milgram, P. and Kishino, F. (1994). A taxonomy of mixed reality visual displays. IEICE Transactions on Information Systems, E77-D(12):1321-1329.

Nurminen, A. (2006). The m-loma mobile 3d map project website. http://www.init.hut.fi/research \%26projects/m-loma/. Last Checked: November, 2008 .

Rademacher, P., Lengyel, J., Cutrell, E., and Whitted, T. (2001). Measuring the perception of visual realism in images. In Proceedings of the 12th Eurographics Workshop on Rendering Techniques, pages 235-248, London, UK. Springer-Verlag.

van Dijk, S., van Kreveld, M., Strijk, T., and Wolff, A. (1999). Towards an evaluation of quality for label placement methods. In Proc. 19th Internat. Cartographic Conf. (ICC'99), pages 905-913, Ottawa, Canada. Internat. Cartographic Association.

Wolff, A. (1999). Automated label placement in theory and practice. In PhD thesis, Freie Universität. 